\title{
FATORES MOTIVACIONAIS PARA PRÁTICA DE ATIVIDADE FÍSICA EM IDOSOS
}

\author{
MOTIVATIONAL FACTORS OF PHYSICAL ACTIVITY PRACTICE IN AIGING \\ PEOPLE
}

Thaís da Silva Nascimento ${ }^{1}$, Lucas Camilo Pereira ${ }^{2}$, Rosane de Almeida Andrade ${ }^{3}$, Thiago Medeiros da Costa Daniele ${ }^{4}$, André Igor Fonteles ${ }^{5}$

\section{RESUMO}

A prática de atividade física é importante para pessoas idosas. É importante compreender os aspectos que podem influenciar o idoso na adesão e na manutenção do estilo de vida ativo e saudável. O objetivo deste estudo é investigar os fatores motivacionais de idosos que praticam atividades físicas regulamente. A coleta de dados foi realizada utilizando-se o IMPRAF-54 (Inventário de Motivação para Prática Regular de Atividades Físicas). A amostra foi composta por idosos com idade entre 60 e 79 anos $(69,3 \pm 5,4), 27(54 \%)$ homens e $19(38 \%)$ mulheres. A maior motivação para atividade física nessa população foi relacionada a saúde $(38,0 \pm 3,7)$ e o fator mais irrelevante foi a competitividade $(9,3 \pm 3,0)$. Uma correlação binária entre os domínios do IMPRAF-54 realizada relacionou que o controle do estresse está positivamente associado à saúde, sociabilidade, estética e prazer. A busca por melhor saúde foi maior motivador para essa população. Algumas diferenças entre os sexos foram encontradas. A competição foi relevante em idosos com idades entre 60 e 69 anos do que idosos com idades entre 70 e 79 anos. Fator motivacional mais importante foi a saúde relacionada à prática de atividade física. A competitividade foi negativamente relacionada à maioria dos domínios relacionados a IMPRAF-54.

Palavras-chave: Motivação; idosos; atividade física; Saúde.

\section{ABSTRACT}

The practice of physical activity is importance for elderly people. It is important to understand the aspects that may influence the elderly in adherence and in maintaining active and healthy lifestyle. The aim of this study is investigate the motivational factors

\footnotetext{
${ }^{1}$ Acadêmica em Educação Física - Centro Universitário UniFanor|Wyden - Fortaleza - CE.

${ }^{2}$ Acadêmico em Educação Física - Universidade de Fortaleza - UNIFOR - Fortaleza -CE.

${ }^{3}$ Mestra em Avaliação das Atividades Físicas e Desportivas - Universidade de Trás-os-Montes e Alto Douro - Vila Real - Portugal - UTAD. Docente do curso de Educação Física - Centro Universitário UniFanor|Wyden - e - Centro Universitário UNIGRANDE - Fortaleza - CE.

${ }^{4}$ Doutor em Ciências Médicas - Universidade Federal do Ceará - UFC - Fortaleza - CE. Docente do curso de Educação Física - Universidade de Fortaleza - UNIFOR - e - Centro Universitário UniFanor|Wyden - Fortaleza-CE.

${ }_{5}^{5}$ Doutor em Saúde Coletiva - Universidade Federal do Rio Grande do Norte - UFRN - (Natal-RN). Docente do Curso de Educação Física - Universidade de Fortaleza - UNIFOR - e - Universidade Federal do Ceará - UFC.
} 
of elderly people who practice physical activities regularly. Data collection was performed using IMPRAF-54 (Inventory of Motivation for Practice Regular of Physical Activities). The sample consisted of elderly people aged between 60 and 79 years (69.3 \pm 5.4$), 27(54 \%)$ men and $19(38 \%)$ women. The highest motivation for physical activity in this population was related health $(38.0 \pm 3.7)$ and the most irrelevant factor was competitiveness $(9,3 \pm 3.0)$. A binary correlation between the domains of IMPRAF54 performed related that stress control is positively associated to health, sociability, aesthetics and pleasure. The search for better health was the greatest motivator for this population. Some differences between genders were found. The competition was more relevant in the elderly aged between 60 and 69 years than elderly aged between 70 and 79 years. The most important motivational factor was health related to practice physical activity mainly for health. Competitiveness was negatively related to most of the IMPRAF-54 related domains of both genders.

Keywords: Motivation; seniors; physical activity; Cheers.

\section{INTRODUÇÃO}

No processo de envelhecimento, podem ser observadas algumas alterações fisiológicas e comportamentais. Comumente se constata o declínio nas capacidades funcionais e, consequentemente, alteração nos aspectos psicológicos envolvidos e decorrentes destas perdas graduais e contínuas pode ainda ser verificada diminuição significativa da atividade motora, resultante, em parte, da degeneração fisiológica, fatores ambientais, doenças, estilo de vida ou a combinação desses elementos ${ }^{1}$.

A prática de atividades físicas em indivíduos idosos, objetiva beneficiar sua autossuficiência. Podendo assim, levar a diminuição dos efeitos deletérios que podem ser observados com o avançar da idade ${ }^{2}$. Sendo assim, a sua prática regular melhora a saúde e a qualidade de vida, levando a uma melhora na capacidade funcional e, consequentemente, desenvolvendo a autonomia e melhora da saúde mental dos idosos $3,4,5$.

Nahas sustenta que, nas sociedades urbanas modernas, os níveis adequados de atividade física e aptidão física, somente são mantidos quando uma forte motivação está continuamente presente, ou seja, quando o indivíduo percebe os benefícios deste comportamento como grande valor para a sua vida, superando as dificuldades para realizar tais ações, e quando as forças sociais oferecem mais facilitadores do que barreiras ${ }^{6}$. Uma metanálise realizada no ano de 2018 afirma que 
a adesão ao exercício pode ser influenciada positivamente; no entanto, fatores adicionais podem ser positivos para alcançar resultados efetivos para a marcha e para o equilíbrio ${ }^{7}$.

Ressalta-se que a motivação para a prática de atividade física, para saúde ou bem-estar é decorrente de uma complexa interação as variáveis psicológicas, sociais, ambientais e até genéticas. Entre os vários fatores destacam-se: o conhecimento, a atitude, o apoio social e familiar, disponibilidade de espaço, sensação de segurança nas vizinhanças e instalações, barreiras percebidas pela pessoa como o tempo, distância, e recursos $8,9,10$.

Foi realizada uma busca na literatura a fim de buscar embasamento teórico, através de estudos realizados, envolvendo a motivação para a prática de atividade física em idosos. O Quadro 1 descreve algumas informações da autoria, ano de publicação, local de realização, instrumentos utilizados e os principais achados. $O$ agrupamento dos estudos foi feito a partir do ano de 2006, buscando compreender a realidade de diferentes localidades.

Diante do exposto, o presente estudo ter por objetivo analisar os aspectos motivacionais para a prática de atividades físicas em idosos fisicamente ativos.

\section{METODOLOGIA}

\subsection{Planejamento da pesquisa}

Trata-se de uma pesquisa quantitativa de caráter transversal em que foi aplicado o Inventário de Motivação para Prática Regular de Atividade Física (IMPRAF54). Os dados foram coletados no período da manhã por um período de dois meses em indivíduos idosos que praticam atividade física regularmente como musculação, treinamento funcional, dança e hidroginástica a mais de um ano. Todos os voluntários assinaram o Termo de Consentimento Livre e esclarecido (TCLE). A aceitação para 
participar do presente estudo foi feita segundo a Resolução 510/20168 Conselho Nacional de Saúde. Resolução n 510, de 07 de abril de 2016 ${ }^{11}$.

\subsection{Local de estudo}

O estudo foi realizado na acessória JC Sport, Casa da Melhor Idade e o projeto Força Viva, localizados na cidade de Aquiraz, Ceará.

\subsection{População e Amostra}

O presente estudo foi realizado com a população de 46 idosos, de ambos os gêneros com idades entre 60 e 80 anos.

Quadro 1. Análise de alguns estudos acerca dos efeitos motivacionais para a prática de atividade física em idosos.

\begin{tabular}{|c|c|c|c|c|}
\hline Autor (ano) & Estado & Amostra & Instrumento & Principais achados \\
\hline Dantas et al ${ }^{12}$ & $\begin{array}{l}\text { Rio de } \\
\text { Janeiro }\end{array}$ & $\begin{array}{l}39 \text { idosos de } \\
\text { ambos os } \\
\text { gêneros ( } \geq 60 \\
\text { anos) }\end{array}$ & IMPRAF-54 & $\begin{array}{l}\text { Principais aspectos } \\
\text { motivacionais descritos pelos } \\
\text { idosos foram: Saúde, seguido de } \\
\text { sociabilidade e prazer. }\end{array}$ \\
\hline Agnes et. al ${ }^{13}$ & $\begin{array}{l}\text { São } \\
\text { Paulo }\end{array}$ & $\begin{array}{c}77 \text { idosos de } \\
\text { ambos os } \\
\text { gêneros } \\
\text { (entre } 55 \text { e } 90 \\
\text { anos) }\end{array}$ & IMPRAF-54 & $\begin{array}{l}\text { O principal fator motivacional foi: } \\
\text { a saúde, em seguida aparecem } \\
\text { empatados sociabilidade, prazer } \\
\text { e controle de estresse. }\end{array}$ \\
\hline Santos et. al ${ }^{14}$ & $\begin{array}{c}\text { Rio } \\
\text { Grande } \\
\text { do Sul }\end{array}$ & 40 idosos & $\begin{array}{c}\text { IPAQ, SF36, } \\
\text { ISSL }\end{array}$ & $\begin{array}{l}\text { Os domínios mais motivadores } \\
\text { para os idosos foram saúde, } \\
\text { socialização e prazer, e os menos } \\
\text { motivadores foram }\end{array}$ \\
\hline
\end{tabular}




\begin{tabular}{|c|c|c|c|c|}
\hline & & & & $\begin{array}{l}\text { competitividades, estética e } \\
\text { estresse. }\end{array}$ \\
\hline $\begin{array}{l}\text { Gomes Júnior } \\
\text { et } \mathrm{al}^{15}\end{array}$ & Ceará & $\begin{array}{l}13 \text { idosos de } \\
\text { ambos os } \\
\text { gêneros ( } \geq 60 \\
\text { anos) }\end{array}$ & $\begin{array}{l}\text { Análise de } \\
\text { conteúdo de } \\
\text { Bardin. }\end{array}$ & $\begin{array}{l}\text { Os idosos mostraram-se } \\
\text { motivados no que se refere aos } \\
\text { efeitos positivos sobre saúde e } \\
\text { socialização. }\end{array}$ \\
\hline $\begin{array}{l}\text { Lins e } \\
\text { Corbucci }{ }^{16}\end{array}$ & $\begin{array}{l}\text { Minas } \\
\text { Gerias }\end{array}$ & 28 Idosos & IMPRAF-54 & $\begin{array}{l}\text { Tem-se o prazer, a saúde e a } \\
\text { sociabilidade como os principais } \\
\text { motivos apresentados. }\end{array}$ \\
\hline Freitas et al ${ }^{17}$ & Recife & $\begin{array}{c}120 \text { idosos de } \\
\text { ambos os } \\
\text { gêneros } \\
\text { (entre } 60 \text { e } 85 \\
\text { anos) } \\
\text { usuários de } \\
\text { dois } \\
\text { programas de } \\
\text { exercícios } \\
\text { físicos }\end{array}$ & $\begin{array}{c}\text { Questionário } \\
\text { adaptado } \\
\text { sobre } \\
\text { motivação } \\
\text { para a prática } \\
\text { esportiva }\end{array}$ & $\begin{array}{l}\text { Os motivos mais importantes } \\
\text { para adesão da prática de } \\
\text { atividade física: melhorar a } \\
\text { saúde, melhorar o desempenho } \\
\text { físico e adotar uma vida mais } \\
\text { saudável. }\end{array}$ \\
\hline Ribero $^{18}$ & $\begin{array}{l}\text { Rio } \\
\text { Grande } \\
\text { do Sul }\end{array}$ & 20 Idosos & $\begin{array}{l}\text { IMPRAF-132, } \\
\text { e } \\
\text { WHOQOLOId } \\
\text { e WHOQOL- } \\
\text { Bref }\end{array}$ & $\begin{array}{l}\text { A saúde apresentou uma maior } \\
\text { media, quando comparada as } \\
\text { outras variáveis de motivação. }\end{array}$ \\
\hline
\end{tabular}

ABREVIATURAS: IMPRAF: Inventário de Motivação para Prática Regular de Atividade Física, IPAQ: Questionário Internacional de Qualidade Física, ISSL: Inventário de Sintomas de Stress para Adultos de Lipp, SF36: Questionário de Qualidade de Vida. 


\subsection{Instrumentos e Procedimentos}

Foi utilizado o Inventário de Motivação para a Prática Regular de Atividades Físicas (IMPRAF - 54). O questionário foi construído e validado por Barbosa ${ }^{19}$. Este instrumento avalia seis dimensões da motivação para a prática da atividade física que são: Controle de estresse, Saúde, Sociabilidade, Competitividade, Estética e Prazer. A validade fatorial (exploratória e confirmatória), bem como a consistência interna foi avaliada $(a>0,80)$ e os resultados obtidos, indicam tratar-se de instrumento válido e fidedigno ${ }^{19}$. Para a análise e interpretação de resultados (escores brutos) foi utilizada a folha de análise e interpretação das respostas IMPRAF-54, na análise e interpretação de resultados (escores percentílicos), foram utilizadas as Tabelas Normativas IMPRAF-54, que transformam os escores brutos em percentis e contextualizar os resultados de acordo com grupo correspondente, permitindo verificar em uma escala de $0 \%$ a $100 \%$, o quanto este indivíduo está motivado para determinada dimensão.

A avaliação e análise de dados deste Inventário aconteceram de acordo com as instruções contidas no Manual Técnico de Aplicação de Barbosa ${ }^{19}$. Sendo ele constituído por 54 itens, agrupados em 9 blocos, que são, de forma individual, avaliados por meio de uma escala Likert de 5 pontos, desde "isso me motiva pouquíssimo" a "isso me motiva muitíssimo".

\subsection{Análise estatística}

Os dados são expressos através de média, desvio padrão e valores percentuais, quando adequado. Foi utilizado o teste de Kolmorov-Smirnof para avaliação da normalidade residual e o teste de Levene para avaliação de igualdade de variância. O teste exato de Fisher foi utilizado entre as variáveis categóricas. Para a comparação entre dois grupos foram utilizados o teste $\mathrm{t}$ de Student ou MannWhitney, quando adequado. Uma análise de correlação binária foi utilizada. Os 
dados foram submetidos a um programa de estatística do SPSS for Windows (SPSS 17).

\section{Resultados}

As análises descritas dos domínios do Inventário de motivação à prática regular de atividades físicas e esportivas (IMPRAF-54) podem ser observadas na Tabela 1. No que concerne aos subitens do IMPRAF-54, a competitividade não se mostrou um dos fatores principais que levam os idosos a praticarem atividades físicas.

Como observado abaixo, foram estudados idosos com idade entre 60 e 79 anos $(69,3 \pm 5,4)$ de ambos os gêneros. Destes, 27 (54\%) homens e 19 (38\%) de mulheres. Totalizando 46 idosos avaliados. Após análise dos domínios do IMPRAF54, constatou-se que a maior motivação para a prática de atividades físicas nessa população se relaciona com a busca pela saúde $(38,0 \pm 3,7)$ e o fator mais irrelevante foi a competitividade $(9,3 \pm 3,0)$.

Tabela 1. Dados descritivos da amostra estudada $(n=46)$.

\begin{tabular}{lcccc}
\hline Variáveis & Mínimo & Máximo & Média & Desvio padrão \\
\hline Idade (anos) & 60 & 79 & 69,3 & 5,4 \\
\hline Controle do estresse & 8 & 40 & 30,3 & 8,2 \\
\hline Saúde & 25 & 40 & 38,0 & 3,7 \\
\hline Sociabilidade & 8 & 40 & 32,5 & 10,5 \\
\hline Competitividade & 8 & 22 & 9,3 & 3,0 \\
\hline Estética & 8 & 40 & 24,2 & 8,8 \\
\hline Prazer & 16 & 40 & 35,4 & 6,2 \\
\hline
\end{tabular}

Uma correlação binária entre os domínios do IMPRAF-54 realizado com todos os voluntários apontou que o controle do estresse se relaciona positivamente com a saúde, sociabilidade, estética e prazer. Na mesma análise, o domínio competitividade se correlacionou negativamente com o controle do estresse (Tabela 2). 


\section{Atenção à saúde}

Tabela 2. Correlação binária entre os domínios do IMPRAF-54 em toda a amostra $(n=46)$.

\begin{tabular}{|c|c|c|c|c|c|c|c|}
\hline & & $\begin{array}{c}\text { Controle do } \\
\text { estresse }\end{array}$ & Saúde & $\begin{array}{c}\text { Sociabilidad } \\
\text { e }\end{array}$ & $\begin{array}{c}\text { Competitividad } \\
\mathrm{e}\end{array}$ & Estética & Prazer \\
\hline Controle do & Pearson & 1 &, $316^{* \star}$ &, $523^{* *}$ &,$- 317^{*}$ &, $303^{*}$ &, $710^{* *}$ \\
\hline estresse & Valor de & & ,032 & ,000 & ,032 & ,041 &, 000 \\
\hline & $p$ & & & & & & \\
\hline Saúde & Pearson & & 1 &, $522^{*}$ &,$- 375^{*}$ & ,298* &, $574^{\star \star}$ \\
\hline & Valor de & & & ,000 & ,010 & ,044 & ,000 \\
\hline & $p$ & & & & & & \\
\hline Sociabilidade & Pearson & & & 1 &,- 159 & ,232 &, $597^{\star *}$ \\
\hline & Valor de & & & & ,291 & , 120 & ,000 \\
\hline & $p$ & & & & & & \\
\hline Competitividade & Pearson & & & & 1 & ,208 &,- 283 \\
\hline & Valor de & & & & & , 166 & 057 \\
\hline & $p$ & & & & & & \\
\hline Estética & Pearson & & & & & 1 & ,443* \\
\hline & Valor de & & & & & & ,002 \\
\hline & $p$ & & & & & & \\
\hline Prazer & Pearson & & & & & & 1 \\
\hline & Valor de & & & & & & \\
\hline & $p$ & & & & & & \\
\hline
\end{tabular}

Ao analisarmos as médias dos domínios do IMPRAF-54 na comparação entre os gêneros, não foram observadas diferenças estatisticamente significante $(p>0,005$; ANOVA) (Figura 1). 
Figura 1. Comparação entre as médias dos domínios quando comparadas entre os gêneros masculino e feminino $(n=46)$.

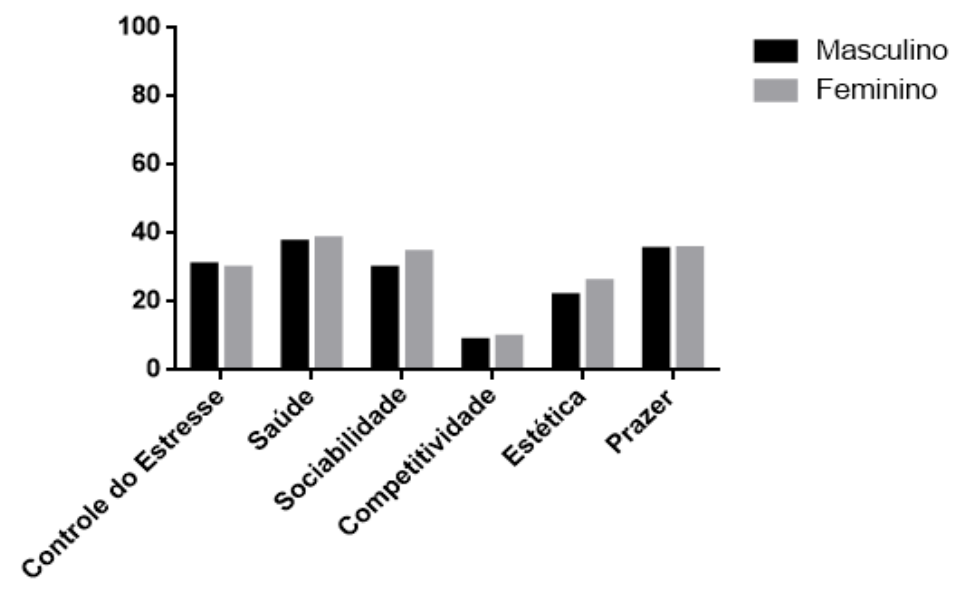

Os idosos com idades entre 60 e 69 anos relataram maior motivação para a prática de atividades físicas quanto à competitividade $(10,2 \pm 3,8)$ quando comparados aos idosos com idade entre 70 e 79 anos $(8,2 \pm 0,6)(p=0,02)$. Dados similares foram encontrados nas análises para o domínio "estética" $(29,9 \pm 7,5$ vs. $21,0 \pm 9,4 ; p=0,02$, respectivamente). Por outro lado, os outros domínios não apresentaram diferenças estatisticamente significantes (Tabela 3 ).

Para uma consistência mais precisa dos resultados, os idosos envolvidos nesse estudo foram separados em dois grupos (Grupo 1= com idade variado de 60 à 69 anos e Grupo 2 = com idade variando de 70 à 79 anos) (Tabela 3).

Tabela 3. Avaliação dos domínios do IMPRAF-54 comparados pela idade dos idosos.

\begin{tabular}{lccc}
\hline Variáveis & $\begin{array}{c}\text { Grupo 1 } \\
(60-69 \text { anos })\end{array}$ & $\begin{array}{c}\text { Grupo 2 } \\
(70-79 \text { anos })\end{array}$ & Valor de $\mathrm{p}$ \\
\hline Controle do estresse & $30,1 \pm 9,2$ & $30,4 \pm 7,1$ & 0,88 \\
\hline Saúde & $38,2 \pm 3,5$ & $37,7 \pm 4,0$ & 0,61 \\
\hline Sociabilidade & $32,4 \pm 9,3$ & $32,5 \pm 12,1$ & 0,98 \\
\hline Competitividade & $10,2 \pm 3,8$ & $8,2 \pm 0,6$ & $0,02^{*}$ \\
\hline Estética & $26,9 \pm 7,5$ & $21,0 \pm 9,4$ & $0,02^{*}$ \\
\hline
\end{tabular}




\begin{tabular}{llll}
\hline Prazer & $36,0 \pm 5,8$ & $34,6 \pm 6,7$ & 0,44 \\
\hline Test T $(p<0,05)$ & &
\end{tabular}

Uma correlação binária foi realizada separadamente por gênero (Tabela 4). Inicialmente, foi constatado que para o gênero feminino houve umA forte correlação positiva entre o controle do estresse e o prazer $(p=0,000)$. Uma relação positiva com a sociabilização e o prazer também foi observada $(p=0,003)$. A competitividade se apresentou com uma relação negativa para a maioria dos domínios relacionados no IMPRAF-54 (Tabela 4).

Tabela 4. Correlação binária entre os domínios do IMPRAF-54 em toda realizada para o gênero feminino $(n=26)$.

\begin{tabular}{|c|c|c|c|c|c|c|c|}
\hline & & Controle do & Saúde & abilidad & mpetitivid & Estética & Prazer \\
\hline & & estresse & & e & ade & & \\
\hline Controle do & Pearson & 1 & ,212 & ,372 &,$- 458^{*}$ & ,222 &, $703^{*}$ \\
\hline estresse & $p$ & & ,297 & ,061 & ,019 & ,275 & ,000 \\
\hline Saúde & Pearson & & 1 &, $747^{* *}$ &,$- 594^{\star \star}$ & ,066 &, $447^{*}$ \\
\hline & $p$ & & & ,000 & , 001 & ,749 & ,022 \\
\hline Sociabilidad & Pearson & & & 1 &,- 384 & ,060 &, $553^{* \star}$ \\
\hline e & $p$ & & & & ,053 & ,771 & ,003 \\
\hline Competitivid & Pearson & & & & 1 & 186 &,$- 440^{*}$ \\
\hline ade & $p$ & & & & & ,363 & ,024 \\
\hline Estética & Pearson & & & & & 1 & 251 \\
\hline & $p$ & & & & & & 216 \\
\hline Prazer & Pearson & & & & & & 1 \\
\hline & $p$ & & & & & & \\
\hline
\end{tabular}

Ao analisarmos o gênero masculino, a relação do controle do estresse com a socialização foi mais forte $(p=0,000)$, a relação com a estética $(p=0,04)$ e com o prazer $(p=0,000)$. Podendo as outras correlações ser observadas na Tabela 5. 


\begin{tabular}{|c|c|c|c|c|c|c|c|}
\hline & \multirow{2}{*}{$\begin{array}{c}\text { Controle de } \\
\text { estresse }\end{array}$} & \multirow{2}{*}{ Saúde } & \multicolumn{2}{|c|}{ Sociabilidad Competitivi } & \multirow[t]{2}{*}{ Estética } & \multirow[t]{2}{*}{ Prazer } \\
\hline & & & & e & dade & & \\
\hline \multirow{2}{*}{$\begin{array}{c}\text { Controle do } \\
\text { estresse }\end{array}$} & Pearson & 1 &, $446^{*}$ &, $722^{\star \star}$ &,- 010 &, $449^{*}$ &, $724^{\star \star}$ \\
\hline & $\mathrm{p}$ & & ,049 & ,000 & ,968 & ,047 &, 000 \\
\hline \multirow[t]{2}{*}{ Saúde } & Person & & 1 & ,315 &,- 089 &, $577^{\star *}$ &, $704^{* \star}$ \\
\hline & $p$ & & & ,176 & ,709 & ,008 & $\overline{001}$ \\
\hline \multirow{2}{*}{$\begin{array}{c}\text { Sociabilidad } \\
\text { e }\end{array}$} & Person & & & 1 & ,044 & ,340 &, $658^{\star \star}$ \\
\hline & $p$ & & & & ,854 & ,142 & ,002 \\
\hline \multirow{2}{*}{$\begin{array}{c}\text { Competitivid } \\
\text { ade }\end{array}$} & Person & & & & 1 & ,207 &,- 023 \\
\hline & $\mathrm{p}$ & & & & & ,380 & ,922 \\
\hline \multirow[t]{2}{*}{ Estética } & Person & & & & & 1 &, $743^{* \star}$ \\
\hline & $p$ & & & & & & ,000 \\
\hline \multirow[t]{2}{*}{ Prazer } & Person & & & & & & 1 \\
\hline & $p$ & & & & & & \\
\hline
\end{tabular}

\section{Discussão}

O presente estudo objetivou analisar os aspectos que influenciam a motivação na prática das atividades físicas em idosos com idade entre 60 e 79 anos de ambos os gêneros. Para isso, foi realizada uma averiguação com o questionário IMPRAF-54 e correlações binárias com toda a amostra e com os dados separados por gêneros.

Observou-se que a média geral dos resultados apontou que a prática da atividade física se relaciona com a busca pela saúde $(38,0 \pm 3,7)$ e o fator mais irrelevante foi a competitividade $(9,3 \pm 3,0)$. Assim, entende-se que a saúde foi 0 principal aspecto motivador da prática de atividade física, seguido das dimensões controle de estresse, sociabilidade, estética e prazer ${ }^{3}$.

No estudo de Cardoso ${ }^{20}$, o resultado das dimensões dos fatores motivacionais alcançou resultados similares aos nossos achados. $O$ fator saúde se mostrou como o principal fator motivacional para a prática de atividade física em idosos ${ }^{3}$. ao aplicar o IMPRAF-54, concluíram que a busca pela saúde também se mostrou como sendo a principal dimensão motivadora, seguida do prazer e sociabilidade. Estudos prévios apontam a saúde como fator primordial para essa população²1,22. 
Grande parte da busca pela saúde se relaciona as mudanças fisiológicas observadas com 0 processo de envelhecimento $\mathrm{e}$, consequentemente, comprometendo a qualidade de vida dessa população ${ }^{23,24}$. Os ganhos oriundos pela prática regular de exercícios físicos proporcionam uma melhora na capacidade motora, funcional e autonomia dos idosos ${ }^{25}$. Com isso, tende a reduzir o aparecimento e/ou complicações oriundas de doenças crônicas não transmissíveis.

No que se refere ao prazer pela prática, Deci e Ryan ${ }^{26}$ argumentam que 0 prazer está relacionado com a motivação intrínseca (interna), em que o indivíduo possui plena satisfação pela atividade, dessa maneira o prazer se torna ainda mais importante para manter o idoso na prática regular da atividade física ${ }^{27}$.

Quando analisados os domínios "estética" e "competitividade", os dados apontam que estas dimensões se tornam menos motivadoras. Alguns autores como Ryan et $\mathrm{al}^{28}$, mostram que a motivação extrínseca, como exemplo os dois domínios citados acima, são de extrema importância para se iniciar a prática. Contudo, para a sua continuidade os fatores intrínsecos são primordiais. Dantas et al ${ }^{11}$ relatou que a competitividade não se torna a motivação mais importante em idosas. Deixando assim em grande clareza que a dimensão "competição" se mostrou pouco importante para a motivação da atividade física. Contudo, no presente estudo os idosos mais novos apresentavam um maior desejo por esse domínio ao comparado com os idosos mais velhos.

Pesquisas relacionadas à prática regular de atividade física em idosos se mostram extremamente importantes, pois como mostra a Teoria da Auto Determinação (TAD), quanto mais autodeterminado estiver um indivíduo mais próximo da motivação intrínseca ele estará, assim mais envolvido ele ficará pois não só a saúde, mas também o prazer pela atividade estarão envolvidos ${ }^{26}$.

Algumas limitações podem ser observadas no presente estudo. Apesar de o questionário utilizado ser útil na detecção da motivação para a prática da atividade física, o número de idosos de mostrou reduzido. Compreende-se que esses achados são úteis na realização de pesquisas futuras acerca do assunto. 


\section{CONCLUSÃO}

A prática de atividade física para idosos é fundamental para manutenção da saúde e funcionalidade dessa população. A compreensão dos aspectos motivacionais se apresenta como fator vital para que possamos manter esse grupo atrelado aos exercícios físicos. A busca pela saúde foi o maior motivador para a prática de atividade física em idosos. No gênero feminino, uma relação positiva com a sociabilização e o prazer também foi observada. E entre os homens a relação do controle do estresse com a socialização foi mais forte, a relação com a estética e com o prazer. A dimensão competição foi a menos importante para essa população.

\section{REFERÊNCIAS}

1. Larsson L, Degens HLiM, Salviati L, Lee Y, Thompson W, et al. SARCOPENIA: AGING-RELATED LOSS OF MUSCLE MASS AND FUNCTION. Am Physiol Soc. 2019;99:427-511.

2. Copeland JL, Good J, Dogra S. Strength training is associated with better functional fitness and perceived healthy aging among physically active older adults : a crosssectional analysis of the Canadian Longitudinal Study on Aging. Aging Clin Exp Res [Internet]. 2018. Available from: http://dx.doi.org/10.1007/s40520-018-1079-6

3. Shepard RJ, Exercício e envelhecimento. Revista Brasileira de Ciência e Movimento. 2003;5(4): 49-56,

4. Simone M, Lucélia B, TÂnia B, Giovana M. ASSOCIAÇÃO ENTRE SINTOMAS DEPRESSIVOS , MOTIVAÇÃO E AUTOESTIMA DE IDOSOS PRATICANTES DE EXERCíCIOS. Rev Bras Ciêmcias do Esporte. 2012;34(3):683-95.

5. Medeiros $\mathrm{T}$, Meireles $\mathrm{V}$, Bruin $\mathrm{S}$ De. Associations among physical activity, comorbidities, depressive symptoms and health-related quality of life in type 2 diabetes. Arq Bras Endocrinol Metab. 2013;57(1):44-50.

6. Nahas MV. Atividade Física, Saúde e Qualidade de Vida: conceitos e sugestões para um estilo de vida ativo. 3 ed. Londrina: Midiograf. 2003

7. Hughes K, Salmon N, Galvin R, Casey B, Clifford A. Interventions to improve adherence to exercise therapy for falls prevention in community- dwelling older adults : 
systematic review and. Oxford Univ Press. 2018. p.1-11.

8. Marina G, João A. Motivação à prática de atividades físicas: um estudo com praticantes não-atletas. Psico-USF. 2010;15(1):125-34.

9. Kosma M, Buchanan D, Hondzinski J. Complexity of Exercise Behavior Among Older African American Women. J Aging Phys Act. 2017;25:333-44.

10. Flannery M. Self-Determination Theory: Intrinsic Motivation and Behavioral Change. Oncol Nurs Forum. 2017;44(2).

11. Brasil. Conselho Nacional de Saúde. Resolução n 510, de 07 de abril de 2016. [acesso em 2016 out. 30]. Disponível em: http://conselho.saude.gov.br/resoluçoes/2016/reso510.pdf.

12. Dantas R, Paz GA, Straatmann VS. Fatores motivacionais de idosos Praticantes do método Pilates. Curso de Fisioterapia, Universidade do Estado do Rio de Janeiro - UERJ, Rio de Janeiro: 2015.

13. Agnes S, Gabriela C, João C, Julia B, Paula F. Fatores motivacionais relacionados à prática de atividades físicas em idosos. Psicol em Estud. 2017;21(4):667-85.

14. Santos SD, Ferenvi DN, Mauhs J, Mascarenhas M. Atividade física como agente motivador em um grupo de idosos pertencentes a um clube esportivo de Porto Alegre. Curso de Educação Física, Centro Universitário Metodista IPA. Porto Alegre: 2015. 15. Júnior VFFG, BRANDÃO, Athus B, ALMEIDA, Francisco JM, Oliveira JGD. Compreensão de Idosos sobre os Benefícios da Atividade Física.19 v.Curso de Fisioterapia, Instituto Superior de Teologia Aplicada. Sobral: 2015.

16. LINS RG, Corbucci PR. Importância da Motivação na Pratica de Atividade Física para Idosos. 2007.Curso de Educação Física, Estação Cientifica Online, Belo Horizonte: 2007.

17. Freitas CMSM, Santiago MS, Viana AT, Leão AC, Freyre C. Aspectos Motivacionais que Influenciam a Adesão e Manutenção de Idosos a Programas de Exercícios Físicos. Curso de Programa Institucional de Bolsa de Iniciação Cientifica, Laboratório de Pratica Desportiva e Lazer, Universidade de Pernambuco, Recide: 2006.

18. Ribeiro JAB. Aspectos motivacionais relacionados à prática de atividade física em idosos e o impacto na qualidade de vida. Programa de Pós-Graduação em Educação Física. Universidade Federal de Pelotas, Pelotas: 2014. 
19. Barbosa ML. Propriedades métricas do inventário de motivação a prática regular de atividade física (IMPRAF-126). Universidade Federal do Rio Grande do Sul. Porto Alegre: 2006

20. Cardoso AMN. Fatores Motivacionais de Idosos Associados à Prática Regular de Exercícios Físicos.122f. Dissertação de Mestrado, Instituto de Biociências, Universidade Estadual Paulista, Rio Claro, SP: 2014

21. Tsujimoto H, Yamada K, Morioka I. Characteristics and Changes in Health Status and Life Function among Female Elderly Participants of Group Exercise. Nihon Eiseigaku Zasshi. 2017;72(1):66-76.

22. Justine M, Azizan A, Hassan V, Salleh Z, Manaf H. Barriers to participation in physical activity and exercise among middle-aged and elderly individuals. J Singapore Med. 2013; 54(10): 581-6.

23. Hao X, Yang $Y$, Gao X, Dai T. Evaluating the Effectiveness of the Health Management Program for the Elderly on Health-Related Quality of Life among Elderly People in China: Findings from the China Health and Retirement Longitudinal Study. J Environ Resarch Public Heal. 2019; 1(16).

24. Wong RMY, Wong $\mathrm{H}$, Zhang $\mathrm{N}$, Chow SKH, Chau WW, Wang J, et al. The relationship between sarcopenia and fragility fracture - a systematic review. Osteoporos Internsational. 2019.

25. Vagetti GC, Filho VCB, Moreira B. Association between physical activity and quality of life in the elderly : a systematic review , 2000-2012. Rev Bras Psiquiatr. 2014; 36: 76-88.

26. Ryan RM, Deci EL. Self-Determination Theory and the Facilitation of Intrinsic Motivation, Social Development, and Well-Being. Am Psychol. 2000;55(1):68-78.

27. Teixeira PJ, Carraça E V, Markland D, Silva MN, Ryan RM. Exercise, physical activity, and self-determination theory: A systematic review. Int J Nutr Physucal Act. 2012.

28. Ryan RM, Frederick C, Sheldon K. Intrinsic Motivation and Exercise Adherence. Int Jounal Sport Psychol. 1997;28:335-54. 